\title{
Demonstrating the value of patient-oriented research in Ontario
}

\author{
Dean Fergusson MHA PhD, Zarah Monfaredi MSc
}

Cite as: CMAJ 2018;190(Suppl 1):S53-54. doi: 10.1503/cmaj.180812

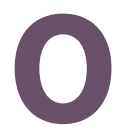

ver the past seven years, the Strategy for PatientOriented Research (SPOR) has been a catalyst of major developments in patient-oriented research practices in Canada. The Canadian Institutes of Health Research (CIHR) created SPOR to provide the evidence needed to inform the development of health policies and improve the health care system. As 1 of 11 units supporting SPOR activity across the provinces and territories, OSSU (Ontario SPOR SUPPORT [Support for People and Patient-Oriented Research and Trials] Unit) provides researchers with the tools to facilitate the conduct of patient-oriented research. In 2014, OSSU put out a call for funding of translational research projects that aimed to improve patient-health outcomes. The OSSU research projects have sought to conduct innovative, measurable, patient-oriented, appropriate, collaborative and transformative research in Ontario; they are known as "IMPACT" demonstration projects. Each IMPACT project had to address a research question aligned with the Ontario Ministry of Health and Long-Term Care priorities as outlined in Patients First: Action Plan for Health Care. ${ }^{1}$ With 17 funded projects in various stages of implementation, we now reflect on the projects' strengths, successes and, importantly, opportunities for improvement.

In the series of commentaries presented in this supplement, recipients of the IMPACT awards reflect on experiences and lessons learned in undertaking patient-oriented research. Paramount is their experience in engaging and involving patients in development of research questions, study design, formulation of interventions, conduct of research and dissemination of results. The OSSU community also acquired an appreciation of the power of innovative study design in patient-oriented research. The IMPACT projects show how novel methods rooted in familiar principles can provide answers to important questions in an efficient and effective way. The emergence of stepped-wedge and cluster crossover designs have the potential to substantially minimize the cost of conducting trials, and greatly enhance the translation and uptake of findings through ensuring their relevance to patients, health care providers and policy-makers. ${ }^{2,3}$ Pragmatic patient-oriented trials prioritize outcomes important to patients, not just those important to providers and the health system. Furthermore, many IMPACT awardees refer to community-based participatory research

\section{KEY POINTS}

- The IMPACT (innovative, measurable, patient-oriented, appropriate, collaborative and transformative) research projects have provided valuable lessons as investigators seek to develop and expand patient-oriented research in Canada.

- The projects have fostered disruptive and innovative study designs and highlighted the importance of multistakeholder collaboration.

- Recipients of the IMPACT awards are calling for improvement of resources available to facilitate patient engagement, including training and guidance in partnering with vulnerable or marginalized populations.

principles, as well as to the familiar "nothing about me without me" statement. Of the studies partnering with Indigenous communities, the First Nations principles of ownership, control, access and possession of data and information ("OCAP") ${ }^{4}$ were implemented, respected and upheld. Rooted in these values, research teams were equipped to conduct innovative pragmatic trials and comparative effectiveness research in areas of priority in partnership with patients.

Another dominant feature demonstrated across IMPACT projects is the importance of collaboration and "team science." Although awarded projects focused on a variety of populations, the common thread was the unmistakable value added by collaborating with patients, health care providers, decision-makers, community members and other key stakeholders. Recipients of the IMPACT awards reported that the outcomes of their research were positively influenced with the inclusion of patient voices. Some studies report the inclusion of patient partners as coinvestigators operating as an equal voice around the table. This exemplifies active and meaningful engagement as we strive for equity across the research process and across stakeholders. ${ }^{5}$ Mechanisms of engagement ranged from patient-led advisory boards, to committees, to community guidance panels, to focus groups. Some IMPACT projects organized collaborative conferences and workshops with the goal of encouraging discussion among key stakeholders and co-developing patient-engagement practices in their particular field. 
The IMPACT projects demonstrate the intuitive: patients are valuable partners and contribute meaningfully in many ways. Across all projects, patient or community partners were engaged in multiple phases of research from conception through to dissemination of findings. To successfully achieve this engagement, several teams included a designated research position - patientengagement facilitator - to support the engagement of patient partners, ease communication between stakeholders, and inform conversations about policy change. Importantly, facilitators also provide the opportunity to help other researchers with patientinvolvement activities and serve as an excellent local resource.

With successes come opportunities for improvement. Recipients of the IMPACT awards are calling for additional resources to meet their needs and set the stage for growth. Pointing researchers to resources, tool kits and examples of best practices is critical, as most are relatively new to the concept and potential value of patient engagement. An example of guidance to equip teams with the tools necessary to conduct meaningful patient engagement is the resource guide created by McMaster University. ${ }^{6}$ This guide summarizes the wide range of evidence relevant to research teams interested in learning more about patient-engagement practice.

Training for patient partners and researchers to learn together and from each other also remains an ongoing effort. The Masterclass on the Use and Conduct of Patient-Oriented Research ${ }^{7}$ is an example of training that embeds the spirit of collaboration and co-learning between stakeholder groups into a foundational curriculum. As we strive for a level of engagement that realizes codesign, co-building and co-leadership, easy access to additional training, expertise and tools at a local level is necessary.

Additionally, ensuring that a range of perspectives is captured among stakeholders has been flagged as an area of difficulty requiring guidance. This is particularly true in research engaging vulnerable and underserviced communities. Whereas many teams were able to nurture strong ties with community groups, others found that guidance for supporting patient partners from a range of backgrounds and experiences is currently lacking. To bridge these gaps, some OSSU-supported centres have established ties with pre-existing hospital patient and family advisory programs (PFAPs). The wealth of knowledge and experience across various health conditions, genders, ages and socioeconomic positions within the PFAP has been important in understanding the barriers to engagement.

The reflections of IMPACT awardees in this supplement have shown the value of patient-oriented research in Ontario. We fully expect each of the projects to have a meaningful impact on the health system and health outcomes owing to the inherent collaborative nature and patient-centredness of the projects. Still, there is much to learn from the experiences of the IMPACT awardees. The Canadian Institutes of Health Research's SPOR and its SUPPORT units, including OSSU, are designed to provide the infrastructure, expertise and support necessary to ensure growth from these experiences. Looking ahead, we expect further innovation and collaboration across the spectrum of patient-oriented research, with the ultimate goal of improving our health system and the health of our population.

\section{References}

1. Patients first: action plan for health care. Toronto: Ontario Ministry of Health and Long-Term Care; 2017. Available: www.health.gov.on.ca/en/ms/ecfa/ healthy_change (accessed 2018 Sept. 13).

2. Hemming K, Haines TP, Chilton PJ, et al. The stepped wedge cluster randomised trial: rationale, design, analysis, and reporting. BMJ 2015;350:h391.

3. Connolly SJ, Philippon F, Longtin Y, et al. Randomized cluster crossover trials for reliable, efficient, comparative effectiveness testing: design of the Prevention of Arrhythmia Device Infection Trial (PADIT). Can J Cardiol 2013;29:652-8.

4. First Nations Information Governance Centre. The First Nations principles of OCAP. Available: https://fnigc.ca/ocapr.html (accessed 2018 Sept. 13).

5. Buck D, Gamble C, Dudley L, et al. From plans to actions in patient and public involvement: qualitative study of documented plans and the accounts of researchers and patients sampled from a cohort of clinical trials. BMJ Open 2014;4:e006400

6. Albelson J. Patient engagement and Canada's SPOR initiative: a resource guide for research teams and networks. Hamilton: McMaster University; 2015. Available: http://ossu.ca/wp-content/uploads/OSSU-Patient-Engagement-Resource -Document-May2015.pdf (accessed 2018 Aug. 10).

7. Masterclass on the use and conduct of patient-oriented research. Toronto: Ontario SPOR SUPPORT Unit; 2018. Available: http://ossu.ca/training/masterclass (accessed 2018 Aug. 10).
More information on this project is available at www.ossu.ca/IMPACTAwards.

Competing interests: Dean Fergusson and Zarah Monfaredi report grants from OSSU (the Ontario SPOR [Strategy for Patient-Oriented Research] SUPPORT [Support for People and Patient-Oriented Research and Trials] Unit), the Canadian Institutes of Health Research (CIHR) and the Ontario Ministry of Health and Long-Term Care.

This article was solicited and has been peer reviewed.
Affiliations: The Ottawa Methods Centre, Clinical Epidemiology Program (Fergusson, Monfaredi), The Ottawa Hospital Research Institute; Faculty of Medicine (Fergusson), University of Ottawa, Ottawa, Ont.

Contributors: Dean Fergusson conceived the direction and foundation for this article. Dean Fergusson and Zarah Monfaredi drafted and revised the article jointly. Both authors gave final approval of the version to be published and agreed to be accountable for all aspects of the work.
Funding: Through OSSU, the Ottawa Methods Centre receives funding from $\mathrm{ClHR}$ and the Ontario Ministry of Health and Long-Term Care.

Correspondence to: Dean Fergusson, dafergusson@ohri.ca 